\title{
Using Coverage to Deploy Formal Verification in a Simulation World
}

\author{
Vigyan Singhal and Prashant Aggarwal \\ Oski Technology, Inc.
}

\begin{abstract}
Formal verification technology has today advanced to the stage that it can complement or replace simulation effort for selected hardware designs. Yet the completion of a formal verification effort is rarely a requirement for hardware tapeout. Simulation remains the primary verification methodology, and means of deciding when verification is complete. In this paper we discuss how formal verification can be deployed using simulation-based coverage in a simulation-based verification schedule.
\end{abstract}

\section{Introduction}

Application-Specific Integrated Circuits (ASICs) enable faster, better, lowerpower products, both for consumer as well as enterprise markets. Competition is fierce. Product lifetimes are shrinking. Meeting project schedule and delivering first-pass silicon, is more important than ever before. Design verification consumes the largest slice of overall design effort in terms of human resources, as much as 60-70 percent [1]. Simulation remains the primary method of pre-silicon verification.

Since its introduction about 20 years ago, formal verification has become increasingly more relevant to a diverse array of applications, widening from custom processor designs to general-purpose ASICs. As with any other new technology, the true test of adoption happens with integration, in this case when formal verification becomes a signoff for chip tapeouts. We are not quite there yet, but that level of adoption is beginning to happen. For formal to become a signoff requirement for tapeout, we should be able to (a) plan for formal verification [2]; and (b) quantitatively measure the results of formal verification, and integrate those with simulation-based measurements. While multiple formal verification methodologies are in practice in the ASIC industry today (e.g., model checking, theorem proving, C-vs-RTL sequential checking), model checking accounts for practically most of the usage, with greater numbers of commercial tools, verification users, as well as licenses in use. Furthermore, major EDA vendors (Cadence, Mentor and Synopsys), as well as a few startups (Jasper, OneSpin and Real Intent) offer competitive solutions. So in this paper, we will focus on the usage of model checking as a formal verification methodology.

The question of when simulation is complete, is often answered using coverage metrics 34. The adoption of formal verification in an ASIC design schedule

G. Gopalakrishnan and S. Qadeer (Eds.): CAV 2011, LNCS 6806, pp. 44 49, 2011.

(C) Springer-Verlag Berlin Heidelberg 2011 
requires formal verification coverage results, using the same metrics as simulation. Several creditable efforts have defined notions of coverage for formal [56]. Simulation-based metrics have also been adopted by formal [7. The key difference in the methodology described in this paper, is that it is based on the practical impossibility of fully and formally verifying all blocks, within typical schedule and resource constraints. Yet, it is imperative that formal verification efforts yield metrics that are integrated with simulation metrics. We will describe a coverage-based methodology that achieves this in practice.

\section{ASIC Verification Process}

Verification is an essential part of the ASIC design cycle. Undetected bugs in any design cause loss of revenue due to delay in time-to-market, and increased nonrecurring engineering cost due to additional re-spins. Well-crafted planning is key for faster verification closure. The selection of the correct verification methods and coverage metrics helps in achieving verification closure faster, and better. The design specification is an input to the RTL implementation (in an HDL), as well as a verification plan (Fig. 1). The verification plan includes choices for the right verification approaches (e.g., formal and simulation), and is based on the scope, required schedule and available resources for each approach. Verification engineers implement their verification setup based on this plan. The verification setup is executed and data is collected for simulation and formal verification runs. The data helps quantify whether planned goals are met or not.

The ASIC management team uses this quantitative data, often in the form of a dynamic verification dashboard (Fig. 2), to track the progress of verification,

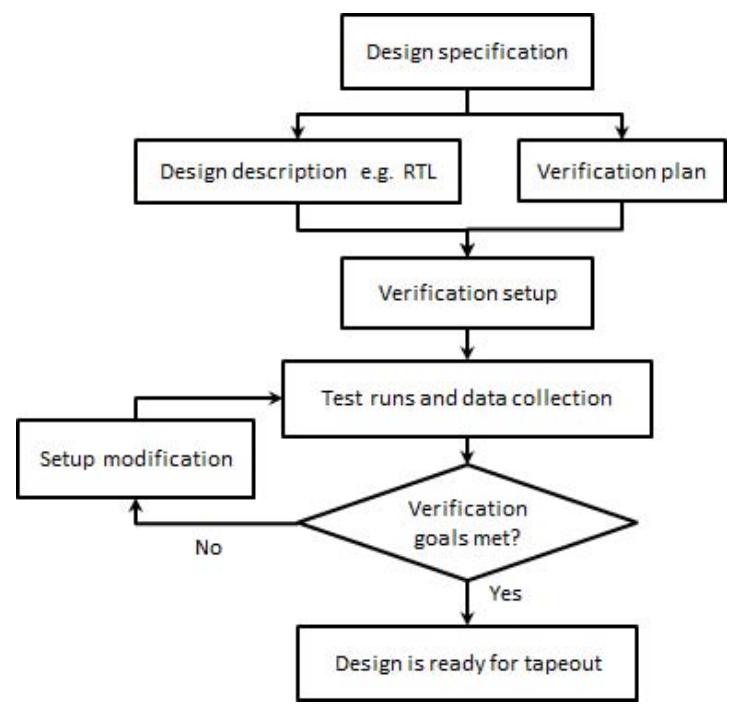

Fig. 1. ASIC verification flow 

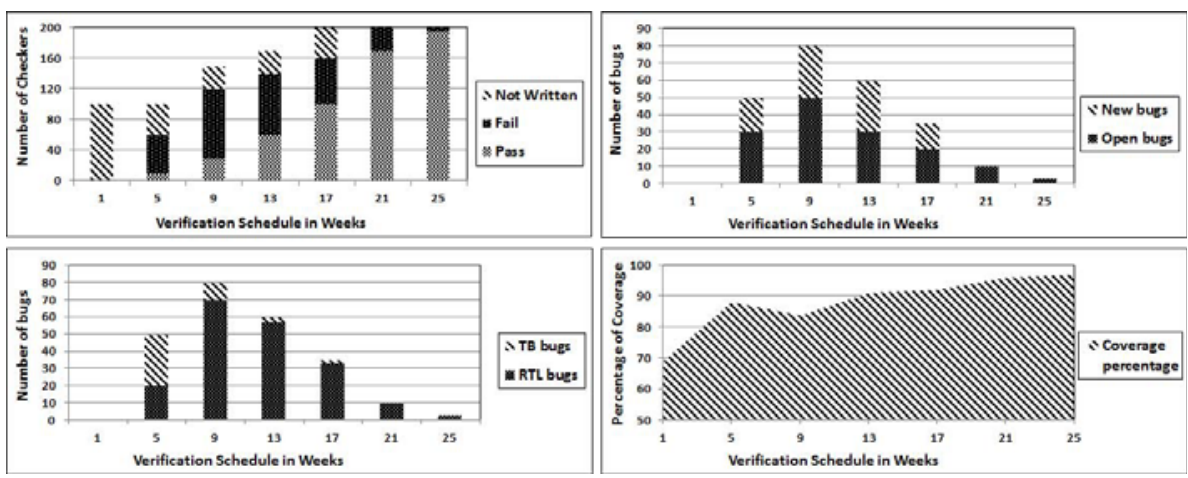

Fig. 2. A verification dashboard

and take timely corrective action. Coverage metrics form an integral part of this progress management.

\section{Coverage Metrics in Simulation}

A simulation testbench setup consists of the following components [8]:

- Bus Functional Models (BFMs), that contain methods used to drive inputs

- Checkers, that monitor inputs, outputs and internals of a design, and flag errors (these checkers could be in-line assertions in the RTL code, external monitors, scoreboard, or even $\mathrm{C}$ reference models)

- Tests, including biases, that control the inputs driven by the BFMs

Coverage is the primary tool used to determine when simulation is complete. The most common coverage metric is code coverage, and line coverage is the most widely used code coverage metric. Line coverage (similar to statement coverage for software testing [9]) computes what percentage of RTL statements were exercised by a given set of tests. For example, consider:

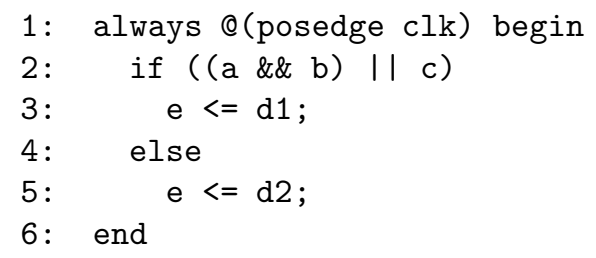

This example results in two line coverage targets, corresponding to lines 3 and 5 . If a test causes c to be 1 , the line 3 will be marked as covered. If no test in a test suite covers line 5 , line coverage for the suite will be reported at $50 \%$. Other variants of code coverage, like expression, branch, toggle, and FSM coverage, are also in use, but are less popular. 
Unlike software testing, $100 \%$ judged line coverage (given, say $99 \%$ automated coverage) is frequently a requirement for an ASIC tapeout - each line that is not automatically reported as covered in simulation, must be manually judged to either redundant or legacy code, or symmetric to another tested line. Tapeout would be delayed until more tests are written to cover the remaining lines. This is typically a higher bar compared to what typical software product releases are exposed to. In spite of the $100 \%$ line coverage requirement, hardware designs are taped out and shipped with bugs, some known and some unknown. Often, meeting the schedule is more important than implementing all the product features that were originally planned. And beating the competition can be even more important. One known limitation of line coverage is that it ignores the observability, or measures the quality of the checkers; we will revisit this in Section 5

Besides code coverage, functional coverage is also beginning to gain acceptance in the ASIC world [8. Due to space limitations, we limit this discussion to code coverage.

\section{Measuring Coverage with Formal Verification}

A formal verification (model checking) environment consists of the following:

- a set of constraints

- a set of checkers (or assertions)

- an optional set of manual abstractions, used to reduce the complexity of formal verification (examples of abstraction methods are data independence abstraction [10] or counter abstraction [11])

The same coverage metrics used in simulation can be applied to answer the question of whether the planned formal verification tasks are complete, or how much the formal verification tasks complement the simulation effort.

Before addressing this topic, we must counter a prevalent myth in formal literature: whether or not bugs are missed in a model checking effort depends solely on how complete the set of checkers is. In practice, this does not hold true - there are other important sources of missed bugs. The most useful model checking efforts end up using bounded model checking (BMC). Furthermore, for reasons of schedule or complexity, the use of (over-)constraints is also very common [2. So bugs may also be missed because the BMC runs did not go far enough; or, because a constraint was an intentional or unintentional overconstraint. BMC is so popular in practice partly because formal verification technology is today able to solve end-to-end verification for complex blocks, especially with the use of manual abstractions.

Given this reality, the simulation-based line coverage metric can be used to mean exactly the same in formal - provided that the constraints used and the bounds reached in BMC (say, $n$ cycles), report what percentage of line targets are reachable in $n$ cycles. For the example in Section 3] if ( ( $\left.\left(\begin{array}{l}\mathrm{a} \& \mathrm{~b} \\ \mathrm{~b}\end{array}\right)|| \mathrm{c}\right)$, in line 2 , is reachable in $n$ cycles, this line would be reported as covered, and otherwise, 
not. Thus, line coverage numbers would mean the same in simulation - whether a certain coverage target is exercised or not. And for formal, this would measure the quality of constraints, as well as the proof bounds reached, perhaps with abstractions. Commercial formal tools are beginning to compute this notion of coverage.

Since we are using the same coverage metrics, we can even merge coverage results. It is often the case that one block is verified end-to-end with formal, and a larger block containing this block is verified with simulation. Even if the line coverage with formal is not $100 \%$ for the block, as long as the unified simulation and formal line coverage is $100 \%$, verification is considered complete from the perspective of line coverage goals. This of course relies on an important assumption - that the set of formal checkers is as complete as the set of simulation checkers. In the next section, we will visit the issue of quantifying the completeness of checkers, both for simulation and formal.

The methodology for integrating formal code coverage results with simulation, can be easily extended to other types of code coverage.

\section{Mutation Coverage}

(Strong) mutation testing applies a set of mutation operators to a design, one at a time, and determines what fraction of mutated designs (called mutants) are exposed by the verification process [12. Mutation testing can result in a code coverage metric (e.g., line coverage), by defining a different mutant for every line of the design, resulting from changing that line. The assignment in every line can, for example, be replaced by an X-assignment, followed by an application of the standard X-propagation-based simulation methodology [13. For the example in Section 3, the mutant for line 3 may look like this:

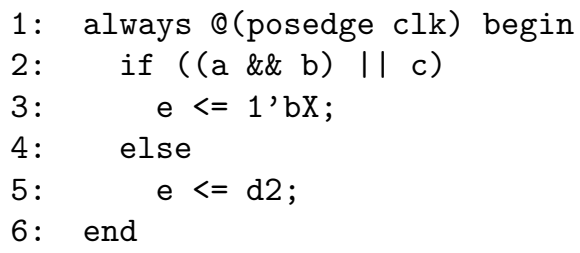

Mutation line coverage is defined as the percent of lines whose mutants will cause some verification test to fail. For example, if the mutant for line 3 is exposed by verification, but the mutant for line 5 is not, we would claim $50 \%$ mutation line coverage.

Clearly, by adding the notion of observability, mutation line coverage becomes strictly more useful than (weak) line coverage described in previous sections. It is accepted in the ASIC verification industry that the latter does not measure the completeness of the checkers, and is a known verification hole. However, mutation coverage is also more expensive to compute.

Along the same lines, model checking tools should be able to implement a feature that measures mutant code coverage, to assess the completeness of the 
formal checkers. For the example code shown above, the line mutant will be achieved by replacing the assignment to e in line 3, by an assignment to new primary input. Of course this will not be an easy metric to compute, given the lines of code in a design. We leave this an important problem that needs to be solved in the future.

In simulation, mutant coverage is often approximated by giving the mutant testing tool a fixed amount of computation resources, and reporting a lower bound on the coverage. The same approach can be adopted for formal. Once we have metrics for both formal and simulation, the results could be combined, and incorporated into the verification dashboard (Fig. 2).

\section{Conclusion}

An optimal verification flow deploys both simulation and formal verification approaches. The choice of common coverage metrics for formal and simulation enables ASIC management teams to integrate formal to complete a comprehensive, planned verification effort.

\section{References}

1. Chayut, I.: Functional verification from a manager's perspective. Synopsys Insight, 1(2) (2006), http://www.synopsys.com/news/pubs/insight/2006/ art1_verinvidia_v1s2.html

2. Foster, H., Loh, L., Rabii, B., Singhal, V.: Guidelines for creating a formal verification testplan. In: Proc. DVCon (2006)

3. Kantrowitz, M., Noack, L.M.: I'm done simulating; now what? Verification coverage analysis and correctness checking of the DECchip 21164 Alpha microprocessor. In: Proc. Design Automation Conf. pp. 325-330 (1996)

4. Tasiran, S., Keutzer, K.: Coverage metrics for functional validation of hardware designs. IEEE Des. Test. 18(4), 36-45 (2001)

5. Hoskote, Y.V., Kam, T., Ho, P.-H., Zhao, X.: Coverage estimation for symbolic model checking. In: Proc. Design Automation Conf. pp. 300-304 (1996)

6. Katz, S., Grumberg, O., Geist, D.: Have I written enough properties? - A method of comparison between specification and implementation. In: Pierre, L., Kropf, T. (eds.) CHARME 1999. LNCS, vol. 1703, pp. 280-297. Springer, Heidelberg (1999)

7. Chockler, H., Kupferman, O., Vardi, M.Y.: Coverage metrics for formal verification. J. Software Tools Technology Transfer 8(4), 373-386 (2006)

8. Bergeron, J.: Writing testbenches using SystemVerilog. Springer, Heidelberg (2006)

9. Myers, G.J.: The art of software testing, 2nd edn. Wiley, Chichester (2004)

10. Wolper, P.: Expressing interesting properties of programs in propositional logic. In: Proc. Symp. Principles Programming Lang POPL, pp. 184-192 (1986)

11. Datta, A., Singhal, V.: Formal Verification of a public-domain DDR2 controller design. In: Proc. VLSI Design, pp. 475-480 (2008)

12. Hampton, M., Petithomme, S.: Leveraging a commercial mutation analysis tool for research. In: Proc. Workshop on Mutation Analysis, pp. 203-209 (2007)

13. Bening, L.C.: Accurate simulation of high speed computer logic. In: Proc. Design Automation Conf. pp. 103-112 (1969) 